\title{
Percepções de um grupo de idosos frente ao fato de não consultarem regularmente 0 cirurgião-dentista
}

\author{
Perceptions of agrap of eddely on thefact of not seeing the dertist regularly
}

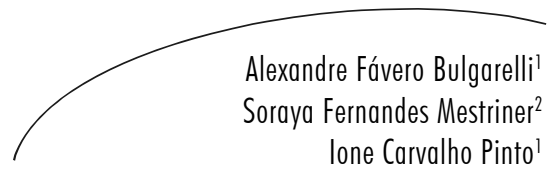

Resumo

Ainda existe no imaginário coletivo a ideia de que idosos não necessitam de assistência odontológica, pois são em sua maioria desdentados e usuários de dentaduras. Assim, a pergunta desta pesquisa é: Qual o motivo que faz com que muitos idosos não consultem regularmente o cirurgião-dentista? O objetivo foi levantar e analisar os motivos que fazem com que idosos cadastrados em uma Unidade de Saúde da Família não consultem regularmente o dentista. Trata-se de uma pesquisa descritiva exploratória de abordagem metodológica quantiqualitativa realizada por meio de entrevistas domiciliares semiestruturadas aplicadas em 149 idosos cadastrados em uma Unidade de Saúde da Família na cidade de Ribeirão Preto,SP. Os dados foram analisados e sistematizados por meio da técnica da Análise de Conteúdo, e para a análise quantitativa foram realizadas análises bivariadas com associações significantes baseadas em valor de $\mathrm{p} \leq 0,05$. Houve associação estatisticamente significante entre visita ao dentista e idade $(p=0,025)$, e observou-se que idosos com idades mais avançadas relataram ir menos ao dentista. Alguns dos motivos alegados foram: medo, dificuldade financeira, falta de tempo, além de falta de sintomatologia dolorosa nos dentes devido ao uso de dentaduras. Concluiu-se que os motivos alegados estão associados a aspectos sociais e culturais que devem ser analisados frente ao cuidado na atenção primária à saúde.

\section{Abstract}

It is still common sense the idea that the elderly do not need dental care because they are largely toothless and denture wearers. Thus the research question is: What is the reason that makes many elderly people do not see the dentist regularly? This study aimed to find and analyze the reason that makes the elderly, enrolled in a family health care center, do not visit the dentist regularly. It is a

Palavras-chave: Saúde bucal. Saúde do idoso. Autopercepção. Atenção Primária à saúde.

Key words: Oral health. Health of the elderly. Self concept. Primary Health Care.

Departamento de Odontologia Preventiva e Social, Faculdade de Odontologia. Universidade Federal do Rio Grande do Sul. Porto Alegre, RS, Brasil.

2 Departamento de Clínica Infantil e Odontologia Preventiva e Social, Faculdade de Odontologia de Ribeirão Preto. Universidade de São Paulo - USP. Ribeirão Preto, SP, Brasil. 
descriptive and exploratory with a quanti-qualitative approach using household semi-structured interviews applied to 149 elderly enrolled at a family Health Unity in Ribeirão Preto city, SP, Brazil. Data were analyzed and standardized by the Content Analysis technique and for the quantitative analysis it was performed bivariate analysis based on $\mathrm{p} \leq 0.05$. There was significant association between dentist visit and age $(p=0,025)$, and it was observed that higher age elderly related going to the dentist few times. Some of the reasons were: fear, financial difficulties, absence of time, and absence of tooth pain and denture wear. It was concluded that these reasons are related to the social and cultural aspects that needs to be analyzed within the primary health care.

\section{INTRODUÇÃO}

Os idosos estão ocupando espaços antes não explorados pela mesma faixa etária por gerações passadas, como academias de ginásticas, universidades, escolas de idiomas, bem como o espaço do exercício profissional. Isso é possível e está acontecendo como consequência da expectativa de vida do brasileiro, que na atualidade firma-se em 73,1 anos de vida e tende a aumentar. ${ }^{1}$

Mais anos vividos com qualidade de vida refletem mudanças no perfil da população que vem envelhecendo lentamente. Mais qualidade de vida está diretamente associada à melhor nutrição, que por sua vez, dentre outros aspectos, é proporcional a uma adequada mastigação que se estabelece de acordo com a presença de dentes e/ ou reabilitações protéticas desempenhando suas funções adequadamente. ${ }^{2}$

Como os idosos estão atingindo idades cada vez mais avançadas, a cavidade bucal deve manterse saudável e desempenhando suas funções por mais tempo, acompanhando a expectativa de vida da população. Dito de outra forma, a saúde bucal tem que acompanhar a expectativa de vida da população, e profissionais da saúde bucal de idosos devem estar atentos a este fato. A promoção da saúde e a prevenção de doenças da cavidade bucal devem estender-se até a velhice e não serem negligenciadas como o eram poucos anos atrás. ${ }^{3}$ Portanto, a adequada manutenção dos dentes e/ ou próteses na cavidade bucal se torna um desafio para a população idosa e para a categoria de profissionais da saúde bucal.
O Brasil, desde 1994, vivencia a tentativa de mudança do modelo de atenção à saúde. Tal mudança vem com a Estratégia de Saúde da Família, estratégia cujo intuito é reorganizar a atenção básica, incluindo assim a saúde bucal na sua agenda assistencial. ${ }^{3}$ É na Estratégia de Saúde da Família que as ações coletivas de saúde bucal encontram espaço para ir ao encontro dos usuários do Sistema Único de Saúde (SUS), sempre tendo como conceito a equidade, levando saúde bucal àqueles que mais necessitam. Neste caso, os idosos são um grupo que deve ser priorizado na atenção à saúde bucal.

Compreendendo a manutenção da saúde bucal como um desafio e algo contínuo para a saúde das populações até a fase da velhice, é necessário buscar constantemente novos sentidos e aspectos relacionados à saúde bucal de idosos. Uma maior atenção à saúde bucal deve sempre ser encorajada nos serviços de saúde e nas universidades, para que as mesmas realizem projetos de educação em saúde e promovam a saúde das pessoas idosas. ${ }^{4}$ Nessa lógica, a visita do idoso ao dentista se torna fundamental para que ações de educação em saúde proporcionem a autopercepção e a autoproteção da saúde, conscientizando as pessoas sobre a necessidade de cuidados com a saúde bucal. ${ }^{5}$

Frente à questão da pesquisa, que está associada à compreensão de certos motivos que fazem com que muitos idosos não consultem regularmente o cirurgião-dentista, o objetivo da presente pesquisa é descrever e analisar as percepções desses idosos frente aos motivos que os fazem não consultar regularmente um cirurgião-dentista de uma Unidade de Saúde da Família. 


\section{MATERIAL E MÉTODO}

Esta é uma pesquisa desenhada metodologicamente por uma abordagem quanti-qualitativa descritiva e exploratória baseada em entrevistas semidirigidas e domiciliares realizadas no ano de 2010, com idosos cadastrados em uma Unidade de Saúde da Família na cidade de Ribeirão PretoSP. Todos os idosos cadastrados na unidade compunham um universo populacional de 512 idosos. Deste universo, 149 idosos afirmaram, em contato prévio com o pesquisador responsável, não consultar regularmente o cirurgião-dentista $\mathrm{da}$ unidade e estes compuseram a amostra intencional $\mathrm{da}$ pesquisa. Os idosos foram entrevistados em suas casas e as entrevista seguiram um roteiro estruturado contendo perguntas como: Por que o Sr(a) não vai regularmente ao dentista da unidade de saúde? O Sr(a) acha importante ter um dentista na unidade, por quê? O Sr(a) pensa ser necessário ir ao dentista da Unidade de Saúde da Família aqui do bairro, por que? As respostas foram gravadas e transcritas para construção do corpus de análise.

Os dados qualitativos foram sistematizados segundo a Análise de Conteúdo, categoria temática que é uma análise transversal que recorta o conjunto das entrevistas por meio de categorias projetadas sobre o conteúdo, e os textos provenientes das falas constituem o corpus para análise. Segundo esta técnica de análise, os dados brutos (textos provenientes das transcrições das falas) passam por um processo de recorte, enumeração e classificação, sendo assim construídas categorias a serem discutidas. Com esta técnica de análise, as leituras iniciais dos discursos (leitura flutuante) vão se tornando precisas em função de hipóteses que vão emergindo em relação ao objetivo e objeto da pesquisa. ${ }^{6}$

Após o tratamento inicial, buscaram-se unidades de registro. Para tal foi utilizado o tema como unidade de registro, no entendimento de que um tema é uma unidade de significação que se liberta naturalmente de um texto analisado, sendo um recorte do sentido da fala, e não uma manifestação formal e regulada. A partir deste momento, chegou-se às categorias de análise que representaram as percepções dos idosos frente à questão da pesquisa. Entendeu-se o processo de categorização como a etapa da análise de conteúdo composta por uma operação de classificação de elementos constitutivos de um conjunto (unidades de registros) por diferenciação e reagrupamento que representa a organização em um sistema de categorias. $^{6}$

Construídas as categorias de análise, as mesmas foram transformadas em indicadores qualitativos, ${ }^{7}$ quantificados e associados às variáveis idade e sexo. Dito de outra forma, as percepções dos idosos foram transformadas em indicadores qualitativos para análise. Em uma contextualização quantitativa e descritiva, a estatística bivariada foi fundamental, pois permitiu a descrição e associação das variáveis com a significância estatística, baseada segundo valor e $\mathrm{p} \leq 0,05$.

Este estudo se enquadra na modalidade de pesquisa de risco mínimo, de acordo com a Resolução n. 196/96 do Conselho Nacional de Saúde, relativa à pesquisa em seres humanos, e teve seu projeto aprovado pela Comissão de Ética em Pesquisa do Centro de Saúde Escola da Faculdade de Medicina de Ribeirão Preto (CEP/ Protocol. 3.245/2009). Cabe observar que a liberdade dos idosos foi imperativa para a realização do estudo e não houve recusas de participação. O consentimento em participar livremente foi estabelecido com a assinatura do Termo de Consentimento Livre e Esclarecido.

\section{RESULTADOS E DISCUSSÃO}

Pode-se afirmar que a população estudada teve maior representatividade feminina, pois dos 149 idosos, 52 (34,8\%) eram homens e 97 (65,2\%), mulheres. Observou-se, portanto, que a maior representatividade da mulher na amostra vem ao encontro de um fato real nas populações mundiais, que é a feminização da velhice, que proporciona uma visão mais feminina das questões abordadas em pesquisas com idosos. ${ }^{8,9}$ No presente estudo, de acordo com a busca por associações estatísticas, não houve diferenças significantes entre as respostas das idosas e dos idosos, o que por sua 
vez estabelece uma relação homogênea entre o ponto de vista dos diferentes gêneros entrevistados.

Em uma ótica da relação com a busca pelo atendimento odontológico, registra-se que em idades mais avançadas os idosos relataram consultar o dentista com menor frequência.
Houve associação estatisticamente significante entre relato de não consultar periodicamente o dentista e idade $(p=0,025)$, onde idosos mais velhos procuram menos o dentista (tabela 1 ). $\mathrm{Na}$ retórica da consulta periódica ao dentista da unidade, constatou-se que os homens e as mulheres têm atitudes semelhantes (sem diferenças estatísticas significantes).

Tabela 1- Não-consulta ao dentista segundo idade. Idosos residentes na área de abrangência de uma Unidade de Saúde da Família. Ribeirão Preto, 2010.

\begin{tabular}{lrrrrrrrrr}
\hline & \multicolumn{2}{c}{ Idade } & \multicolumn{2}{c}{70 a 69} & \multicolumn{2}{c}{$80 \mathrm{e}+$} & \multicolumn{2}{c}{ Total } \\
Não Consulta & $\mathrm{n}$ & $\%$ & $\mathrm{n}$ & $\%$ & $\mathrm{n}$ & $\%$ & $\mathrm{n}$ & $\%$ \\
\hline \multirow{2}{*}{} & & & & & & & & & \\
Masculino & 18 & 34,6 & 27 & 51,9 & 7 & 13,5 & 52 & 100 \\
Feminino & 41 & 42,2 & 45 & 46,4 & 11 & 11,4 & 97 & 100 \\
\hline
\end{tabular}

Segundo Salles, ${ }^{10} 87,0 \%$ dos idosos de seu estudo não procuravam assistência odontológica com frequência, dado que se assemelha ao presente estudo.

Ainda em uma contextualização quantitativa, os motivos que emergiram das categorias após a sistematização dos dados foram descritos e quantificados segundo seus temas. Pode-se observar que a grande maioria $(67,78 \%)$ dos idosos relatou não consultar o dentista, por acreditar que possuindo prótese total (dentadura) não era necessário procurar assistência odontológica regularmente. Fato curioso foi que $6,7 \%$ dos idosos relataram não consultar o dentista por falta de costume/hábito, colocando os cuidados com a saúde bucal em uma posição de necessidade rotineira sem importância. Ou seja, caso a visita ao dentista fosse um hábito presente no seu estilo de vida, o idoso frequentaria regularmente o dentista. Deste modo, o desafio para os profissionais da saúde é criar uma condição de empoderamento desses idosos, para incorporarem em suas atividades da vida diária os cuidados com a saúde bucal.

Embora pequena, houve uma parcela de idosos $(4,72 \%)$ que relatou não consultar o dentista por medo, e o restante da amostra (31 sujeitos) relatou falta de tempo e dinheiro para ir ao dentista. Todos esses dados estão representados na figura 1 . Registra-se que nesta população de idosos, questões sociais, culturais, de estilo de vida, bem como questões econômicas, permitem surgir barreiras que impedem o acesso à atenção, assistência e cuidado com a saúde bucal, mesmo dentro de um modelo de atenção focado na Atenção Primária à Saúde. 


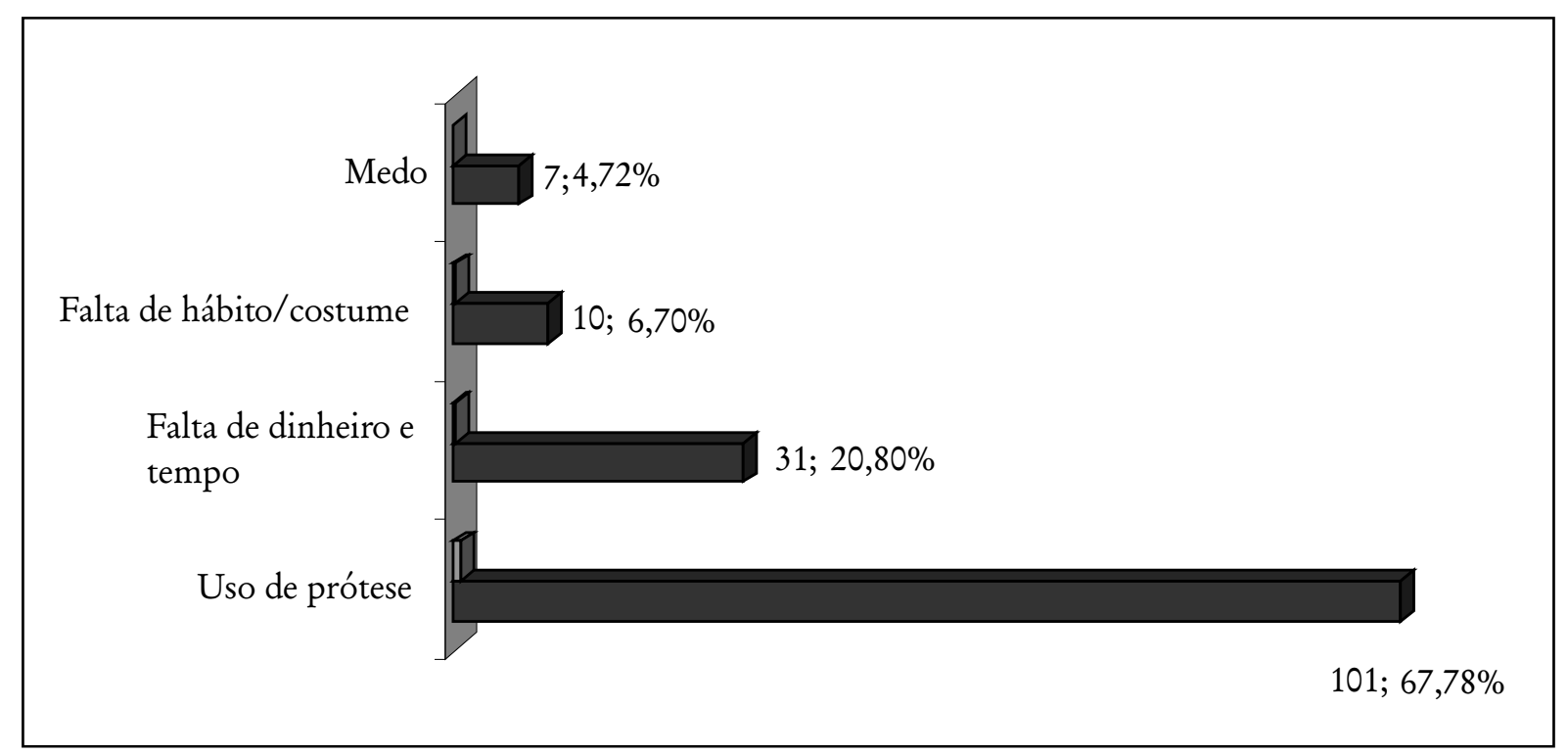

Figura 1 - Motivos referentes ao fato de não consultar regularmente o dentista. Idosos residentes na área de abrangência de uma Unidade de Saúde da Família. Ribeirão Preto, 2010.

Os conceitos de acesso, acolhimento e humanização são de fundamental importância quando se refere a estudos desenvolvidos no contexto da Atenção Primária à Saúde. ${ }^{11}$

Segundo as premissas da Atenção Primária a Saúde, bem como a Política Nacional de Atenção Básica, a saúde bucal é condição básica e essencial para uma adequada saúde geral do indivíduo. ${ }^{11,12}$ Esses contextos estão relacionados com a estrutura multidisciplinar da equipe de saúde da família, que por sua vez garante o acesso aos cuidados básicos e ao acolhimento da população adscrita no que tange à informação a saúde e humanização do atendimento.

Dentro desse contexto, considera-se que a Unidade de Saúde da Família do presente estudo contém, em sua equipe mínima, um cirurgiãodentista que desempenha sua função embasada nos conceitos supracitados. Esse profissional realiza atendimento clínico por demanda espontânea e programada, bem como visitas domiciliares para assistência aos idosos acamados, quando necessário.

Na presente pesquisa, idosos acamados foram excluídos da amostra, pois não se questiona a disponibilidade de acesso ao serviço de saúde bucal da referida Unidade, mas o motivo relatado pelo idoso por não procurar atendimento odontológico na mesma.

Em uma abordagem qualitativa, as categorias que no presente estudo refletem os motivos da não-consulta ao cirurgião dentista, representam as percepções desses idosos frente à relação saúde bucal/idoso. Baseando-se nesta premissa, foram construídas as categorias (motivos) que refletem por que os idosos não percebiam a consulta ao dentista como um ato importante e que deve ser realizado regularmente. Desta forma, os motivos alegados pelos idosos serão apresentados e discutidos distintamente, pois constituem quatro eixos temáticos para discussão.

\section{Falta de tempo e dinheiro}

Compreende-se o acesso como um conceito ampliado de busca pela atenção à saúde. De certo modo, o acesso à saúde, conceito que vem suportar a importância da Estratégia de Saúde da Família como a porta de entrada preferencial para a assistência a saúde no SUS, envolve algumas situações que por vezes se refletem em diversos 
discursos dos usuários. Neste estudo, a dificuldade financeira e a falta de tempo se mostraram como barreiras para o acesso ao serviço de saúde bucal ofertado na referida unidade.

Segundo Azevedo, ${ }^{13} \mathrm{o}$ aspecto organizacional de uma Unidade de Saúde da Família é determinante no acesso da população à saúde, visto que muitas vezes o modo de agendamento das consultas, o modo de acolhimento, a demora no retorno dos resultados dos exames laboratoriais e o excessivo número de famílias por equipe dificultam a identificação da unidade de saúde com a comunidade em que atua. Esta falta de identidade serviço-comunidade faz com que de certa forma haja desinformação sobre o funcionamento da mesma em relação à porta de entrada para um serviço universal e integral.

No mundo pós-moderno, início do século XXI, muitos são os idosos que participam da vida orçamentária e educacional da família desempenhando suporte financeiro e de cuidado. Dentro de um contexto de atenção baseado no ciclo familiar, ${ }^{14}$ verifica-se que muitos idosos moram com os filhos e assim participam do cuidado e educação direta dos netos. Ou seja, as famílias que antes eram constituídas por pai, mãe e filhos, na atualidade iniciam um processo de inclusão dos avôs e das avós.

As famílias ciclam com a presença de filhos pequenos e idosos simultaneamente. Deste modo, devido ao aumento da longevidade, encontramse famílias com três, quatro e até cinco gerações convivendo em um mesmo lar. ${ }^{15}$ Este fato faz com que os idosos muitas vezes não encontrem tempo para buscar informação sobre o autocuidado com a saúde, pois estão voltados aos cuidados e educação dos netos, o que consome seu tempo livre e muitas vezes suas rendas.

Neste contexto, estudo realizado por Hiramatsu et al. ${ }^{16}$ mostra que a falta de conhecimento e autopercepção sobre a necessidade de cuidados odontológicos em muitos casos acarreta dificuldade de acesso ao cirurgião-dentista. É essencial entender que a maneira como uma pessoa idosa percebe sua condição de saúde bucal, bem como o acesso ao serviço de saúde, condiciona a maneira, a importância e o comportamento frente às ações de cuidado com a saúde bucal. ${ }^{5}$

Outro aspecto observado com a construção deste eixo para discussão é a dificuldade financeira. Acredita-se que, muitas vezes, a dificuldade financeira reflete dificuldade de acesso ao cirurgiãodentista. Ressalta-se também que muitos idosos compartilham a percepção cultural e coletiva de que ir ao dentista custa muito caro. Esses mesmos idosos desconhecem a saúde como um direito de todo o cidadão e dever do Estado, e tal desconhecimento leva à não-procura pelo dentista, pois não há diferenciação entre o modelo de atenção à saúde do sistema público de saúde e a odontologia mercantilista privada. Frente a este aspecto, para a população estudada, alguns idosos não percebem que fazem parte de uma população cadastrada no sistema de saúde pública e que têm acesso ao cuidado com a saúde bucal por meio da Estratégia de Saúde da Família.

Foram encontrados na literatura estudos que compartilham semelhantes percepções, visto que a dificuldade de acesso de muitos idosos aos serviços de saúde existe em função da pobreza material revelada por aspectos que vão desde a falta de condução para ir ao posto de saúde até falta de dinheiro para comprar as medicações prescritas. ${ }^{17,18}$ Sabe-se também que, de acordo com o que se encontra na literatura, para muitos idosos a dificuldade financeira constrói a percepção de que o acesso ao cirurgião-dentista é dificultado, e assim ao visitar o cirurgião-dentista esse fato estimula a busca pela simples extração dentária. ${ }^{19}$ Tais evidências científicas são congruentes com o presente estudo.

\section{Falta de hábito/costume de ir ao dentista}

Sabe-se que a consulta ao cirurgião-dentista na maioria das vezes acontece devido à necessidade de sanar alguma dor ou desconforto na cavidade bucal. No caso de não existir incômodo ou dor, dificilmente se busca assistência odontológica para prevenção, ou seja, não existe o costume de ir periodicamente apenas para acompanhamento da saúde bucal. Neste contexto, observa-se que 6,7\% 
dos idosos entrevistados alegaram não frequentar o cirurgião-dentista por não estarem habituados a essa atividade.

Acredita-se que o cirurgião-dentista está no processo incipiente de ser reconhecido enquanto profissional da saúde diretamente relacionado à qualidade de vida das pessoas e que o usuário o associe a uma busca frequente para cura e prevenção de doenças da cavidade bucal e promoção de saúde. Esse reconhecimento se torna real no momento em que a saúde bucal entra para a agenda de saúde das Equipes de Saúde da Família dentro da Atenção Primária à Saúde. ${ }^{3}$

Como destacado no presente tema, que constitui um dos pilares do conteúdo das falas dos idosos, alguns idosos declararam não se preocupar em visitar o cirurgião-dentista, pois não estão habituados a realizar tal atividade. Observa-se que é necessário construir o hábito para que a visita ao cirurgião-dentista se torne um processo natural do cuidado com a saúde bucal. O hábito de visita ao dentista é construído com o empoderamento da comunidade, para que haja o autocuidado e, assim, a busca de atenção à saúde bucal.

O hábito de ir ao dentista não foi muito comum na população do estudo de Strayer et al., ${ }^{20}$ segundo o qual apenas $7 \%$ de idosos tinham o costume de visitar o dentista regularmente. A literatura mostra que com o passar da idade a busca de serviços odontológicos diminui, ao contrário da busca por médicos, que aumenta. ${ }^{20}$

Em seu estudo, Almeida et al. ${ }^{3}$ destacaram que mais de $82 \%$ dos idosos analisados relataram não ir ao dentista regularmente pois não tinham esse costume. No presente estudo, esse relato foi proporcionalmente menor, sendo referido por apenas dez entrevistados, correspondendo a $6,7 \%$ do total daqueles que não iam ao dentista. É interessante registrar que o elevado número de idosos desdentados que não buscam o serviço de saúde bucal -reduzindo assim a necessidade de tratamento odontológico - pode estar associado à baixa expectativa do idoso em relação à intervenção assistencial realizada pelo dentista. ${ }^{21,22}$
Paralelamente a este fato, há uma deficiência de informação aos idosos brasileiros quanto aos benefícios da reabilitação e higienização bucal, bem como a importância das consultas periódicas ao cirurgião-dentista. ${ }^{23}$ Portanto, a literatura vem ao encontro do presente estudo quando o motivo da não-consulta regular ao dentista se mostra uma realidade entre muitos idosos brasileiros.

Uso de prótese total e falta de sintomatologia dolorosa

Durante muitos anos, o sistema de saúde pública brasileiro tinha na assistência à saúde bucal um modelo excludente, mutilador e focado na reabilitação de morbidades da doença cárie. Gerações de brasileiros que eram usuárias do sistema público de saúde sofreram com a extração dentária, com a falta de procedimentos preventivos coletivos e de informação sobre cuidados como a saúde bucal. Como consequência, há hoje em dia idosos com altos índices de edentulismo e necessidade de uso de próteses dentárias.

Se fizermos uma associação cronológica, temos que jovens e/ou adultos que sofreram no passado com mutilação dentária hoje são idosos que muitas vezes carregam consigo a ideia de que, por não possuírem mais dentes naturais, não se encontram no escopo de pessoas que necessitam frequentar regularmente o cirurgião-dentista. A assistência odontológica do passado deixou a imagem, para certos idosos, de que a odontologia é necessária apenas para realizar procedimentos em relação a um órgão, o dente. Esse modelo de assistência não foi capaz e não se preocupava em sensibilizar seus usuários - adultos e idosos de hoje - a contextualizarem "o dente" em um sistema composto por outros órgãos que também necessitam de cuidados, gerando uma consciência coletiva de cuidado e visitas frequentes ao cirurgião-dentista para prevenção doenças e promoção de saúde bucal.

Dentro deste enfoque, verifica-se que a falta de sintomatologia dolorosa no dente e o uso de próteses totais, relatados por $101 \mathrm{idosos}(67,7 \%)$ não estiveram associadas ao sexo e à idade. Tais 
condições foram relatadas pela maioria dos idosos e refletiam seus próprios pontos de vista, construindo o entendimento de que a falta de dente e o uso de próteses totais os desvinculavam de qualquer necessidade de cuidados preventivos e de acompanhamento de doenças e/ou situações incômodas na manutenção da saúde bucal. Para esses idosos, não havendo dente natural e sendo portador de dentadura, não haveria necessidade de consultar o dentista, pois a concepção de saúde bucal construída ao longo de experiências provenientes de um modelo mutilador a simbologia do dentista está relacionada apenas com extração de dentes e substituição por próteses totais.

O uso de prótese total revela-se como um motivo pela não-procura ao cirurgião-dentista $\mathrm{e}$ muitas vezes, para certos idosos, o uso de próteses totais é percebido como um fato de que os idosos estão em desvantagem em relação àqueles que possuem dentes naturais. ${ }^{24}$ Estudo de Weyant et al. ${ }^{25}$ mostrou que $93,6 \%$ dos idosos analisados declararam não ir ao dentista, e relataram o fato de possuírem dentadura como motivo pela nãoconsulta odontológica periódica.

Muitos idosos não buscam a assistência odontológica, pois acreditam que visitar regularmente o dentista é necessário apenas para as pessoas que possuem dentes. Os idosos com essa concepção acreditam, então, que ir ao dentista não é necessário. ${ }^{21,22}$ Tais aspectos sustentam a discussão e os resultados encontrados no presente estudo.

\section{Medo de ir ao dentista}

Uma pequena parcela de idosos entrevistados relatou medo das ações desempenhadas pelo dentista. Na busca pelo entendimento deste fato, foi possível entender que certos idosos mesclaram a percepção de medo com experiências odontológicas desagradáveis vivenciadas no passado, o que os impedia buscar atendimento odontológico. Acredita-se que tais situações fizeram com que esses sujeitos construíssem percepções negativas frente ao tratamento odontológico.
Em muitos casos, a percepção negativa do tratamento odontológico é proveniente de um passado em que a prática odontológica era associada a tortura, sofrimento e dor. Essas percepções fazem com que o dentista tenha uma simbologia, ainda nos dias atuais, de profissional que causa dor.

O medo do tratamento odontológico é chamado na atualidade de "odontofobia", e tem sua genealogia nas ciências psicossociais e comportamentais. Designa uma condição emocional em que é necessária uma abordagem diferenciada ao paciente, pois o mesmo sente ansiedade frente ao tratamento odontológico, o que o impede de visitar o dentista. O medo das pessoas de irem ao dentista está muitas vezes associado ao fato de possível falta de autocontrole no momento em que o usuário do serviço se imagina passando novamente por experiências de dor e sofrimento. ${ }^{26}$

Ao analisar o medo em associação ao tratamento odontológico, compartilha-se do pensamento de Cesar et al., ${ }^{27}$ que afirmam que o medo é frequentemente referido como motivo para a não-realização de consulta odontológica. Além deste aspecto, baixas taxas de utilização de serviços odontológicos por idosos podem ser influenciadas pelo medo de tratamento

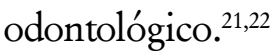

$\mathrm{Na}$ tentativa de compreender o discurso dos idosos, tem-se em mente que muitos de seus contextos são provenientes de sua socialização primária. Desta forma, esta pesquisa se fundamentou em estudos que demonstram que experiências odontológicas na infância e na juventude são fundamentais neste contexto. Essas experiências, quando associadas a sensações de dor, contribuem decisivamente para formar o sentimento de medo e posteriores resistências à consulta odontológica em idades mais avançadas. ${ }^{26}$ Associar as experiências traumatizantes com mensagens vinculando-as à punição por comportamento inadequado na infância parece contribuir também para agravar o sentimento de medo em idades mais avançadas. ${ }^{26-28}$ 
Dor e medo são sentimentos estritamente ligados à imagem do dentista, ainda que se reconheça uma evolução na odontologia, tanto em relação aos equipamentos e tecnologias, quanto à formação e conduta do profissional. $\mathrm{O}$ medo esteve presente, nas falas de alguns idosos, fortemente associado à imagem do contexto de trabalho do dentista. Esse sentimento faz parte do cotidiano da relação dentista-paciente, aparecendo nos relatos tanto como uma percepção do entrevistado, como de seu mundo social. Verifica-se, dessa forma, que o medo não é hegemônico, mas existe.

\section{CONSIDERAÇÕES FINAIS}

A busca pelo serviço odontológico na velhice é necessária para que os idosos possam controlar sua saúde bucal e manter sua qualidade de vida. O presente estudo levantou diferentes percepções relacionadas aos motivos que fazem com que idosos não procurem regularmente o dentista. Tais percepções se articulam e permitem construir sentidos para a não-visita ao dentista.

Alguns idosos, mesmo cadastrados na área de abrangência de uma unidade de saúde da família, afirmam precisar de dinheiro para cuidar da saúde bucal, o que mostra seu desconhecimento sobre a saúde como direito de todo cidadão e dever do Estado. É necessário informar diretamente ao idoso que no SUS não é preciso gastos financeiros para prevenir doenças bucais, tratar de problemas da boca e reabilitar as funções da cavidade bucal.

Aspecto importante a ser considerado é que unidades de saúde da família devem trabalhar questões de acesso ao serviço de saúde bucal dos idosos de acordo com a realidade da comunidade local. A realidade de muitos territórios de saúde é a presença de muitas famílias em cujos núcleos familiares convivem três gerações (filhos, avós e netos). Seria interessante criar condições de acesso dos avós, juntamente com seus netos, na assistência e no cuidado a saúde bucal, despertando assim o interesse do idoso para o cuidado com a saúde bucal e tendo como interlocutores seus netos.

É necessário também trabalhar a educação em saúde, para que os idosos sejam conscientizados da necessidade de encontrar tempo para cuidar da saúde bucal, frequentando regulamente a Unidade de Saúde da Família em busca de informações para cuidar da saúde bucal.

Quanto à outra percepção construída no presente estudo, entende-se que é necessário trabalhar, na condição de profissionais da saúde, diferentes maneiras de enfrentar a odontofobia em idosos. Fica a sugestão de identificar a odontofobia na população idosa e organizar projetos terapêuticos de acordo com a singularidade de cada idoso, para a compensação do não-cuidado devido ao medo de ir ao dentista. Assim, fica a sugestão de avançar, na condição de profissionais da saúde, na tentativa de mudar algumas dessas percepções dos idosos para que ocorra a promoção saúde bucal.

De certa forma, é na Estratégia de Saúde da Família, por meio de um modelo de atenção universal, que existe espaço para mudar o significado construído socialmente de que o dentista é um profissional mutilador e que causa sofrimento e medo ao idoso. 


\section{REFERENNCIAS}

1. Instituto Brasileiro de Geografia e Estatística / IBGE. Em 2003, expectativa de vida do brasileiro subiu para 71,3 anos. [ Acesso em 10 mar 2011. Disponível em: http://www.ibge.gov.br/home/ presidencia/noticias/

noticia_visualiza.php?id_noticia $=266$. Acesso 10.03.2011.

2. Caldas Júnior AF, ,et al. O impacto do edentulismo na qualidade de vida de idosos. Rev. Ciênc. Méd 2005; 14(3):229-238.

3. Brasil. Ministério da Saúde. Secretaria de Atenção à Saúde.. Diretrizes da Política Nacional de Saúde Bucal. Brasília: MS; 2004.76 p.

4. Almeida MEL, Moimaz SAS, Garbin CAS, Saliba NA. Um olhar sobre o idoso: estamos preparados? R. Fac. Odontol. Porto Alegre 2004; 45(1):64-68.

5. Silva SRC, Fernandes RAC. Autopercepção das condições de saúde bucal por idosos. Rev Saúde Pública 2001; 35:349-55.

6. Bardin L. Análise do conteúdo. Lisboa: Editora 70; 1994

7. Minayo MCS. Construção de Indicadores qualitativos para avaliação de mudanças. Rev Bras Educação Médica 2009; 4(33):83-91.

8. Salgado CDS. Mulher idosa: a feminização da velhice. Estud. interdiscip. envelhec 2002;1(4):7-19.

9. Camarano A. Envelhecimento da população brasileira: uma contribuição demográfica.[ Acesso em 03 fev 2011]. Disponível em http:// www.alzheimer.med.br/demografia.pdf. Acesso dia 03.02.2011.

10. Salles AES. Estudo populacional relacionando saúde geral, uso de medicamentos, condutas, hábitos e tempo de uso de prótese total em idosos desdentados totais na cidade de Ribeirão Preto, Ribeirão

Preto.Dissertação(Mestrado em Odontologia) Universidade de São Paulo;2002

11. Starfield B. Primary care: balancing health needs, services and technology. Oxford: Oxford University Press; 1998. 438 p.

12. Brasil. Ministério da Saúde. O SUS pode ser seu melhor plano de saúde. 3. ed. São Paulo: IDEC; 2006.76 p.

13. Azevedo ALM. Acesso à atenção à saúde no SUS: o PSF como (estreita) porta de entrada. Recife.. Dissertação (Mestrado em saúde pública) - Centro de Pesquisas Aggeu Magalhães. Fundação Oswaldo Cruz; 2007
14. Moysés ST, Kriger L, Moysés SJ, Saúde bucal das famílias: trabalhando com evidências. São Paulo: Editora Artes Médicas; 2008. 308 p.

15. Lehr U. A revolução da longevidade: impacto na família, sociedade e individuo. Est. Interdiscipl. Envelhec. 1999; 1(12):7-36.

16. Hiramatsu AA, Tomita NE, Franco LJ. Perda dentária e a imagem do cirurgião-dentista entre um grupo de idosos Ciênc. saúde coletiva 2007;12(4):123-127.

17. Reis SCGB, Marcelo VC. Saúde bucal na velhice: percepção dos idosos, .Ciênc. Saúde Coletiva 2006;11(1):191-199.

18. Moreira TP, Nuto SAS, Nations MK. Confrontação cultural entre cirurgiões-dentistas e a experiência de usuários de baixa renda em Fortaleza Saúde em Debate 2004; 28(66):58-67.

19. Silva MES, Magalhães CS, Ferreira EF. Perda dentária e expectativa da reposição protética: estudo qualitativo. Ciênc. Saúde Coletiva 2010; 15(3):813-820.

20. Strayer MS, Kuthy RA, Caswell RJ, Moeschberger ML. Predictors of dental 13. use for low-income, urban elderly persons upon removal of financial barriers. Gerontologist 1997; 37(1): 110-16.

21. Locker $\mathrm{D}$,, et al.Utilization of dental services by older adults in four Ontario communities. J Can Dent Assoc 1991; 57(11): 879-86.

22. Barros AJD, Bertoldi AD. Desigualdades na utilização e no acesso a serviços odontológicos: uma avaliação em nível nacional. Ciênc. Saúde Colet 2002; 7(4): 709-17.

23. Monti LM, et al.Análise comparada da saúde bucal do idoso na cidade de Araçatuba. Rev. Bras. Geriatr. Gerontol.2006; 9(2):120-125.

24. Jones JA, Orner MB, Spiro A, Kressin NR. Tooth loss and dentures: patient's perspectives. Int Dent J 2003; 53(5):327-334.

25. Weyant RJ, Pandav JL, Plowman JL, Ganguli M. Medical and cognitive correlates of denture wearing in older community-dwelling adults. J. Am. Geriatr. Soc 2004; 52(4):596-600.

26. Skaret E, Soevdsnes EK. Behavioural science in dentistry: the role of the dental hygienist prevention and treatment of the fearfull dental patient. International J Dental Hygiene 2005;3(1):2-6.

27. Cesar LCG, Narvai PC, Gattás VL, Figueiredo GM. "Medo do Dentista" e 
demanda aos serviços odontológicos em municípios da zona oeste da região metropolitana de São Paulo. Odontologia e Sociedade 1999; 1(1/2): 39-44.
28. Oliveira JA, Ribeiro EDP, Bonachela WC, Capelozza ALA. Perfil do paciente odontogeriátrico da Faculdade de Odontologia de Bauru . PCL 2002;4(17):71-79.

Recebido: 21/3/2011

Revisado: $28 / 11 / 2011$

Aprovado: 02/12/2011 
